Slavica

bruxellensia

\section{Slavica bruxellensia}

Revue polyphonique de littérature, culture et histoire

slaves

$1 \mid 2008$

Théâtre

\title{
La mythification des professions dans la culture traditionnelle des Slaves de l'Est
}

\author{
Aleksey V. Yudin \\ Traducteur : Anne Delizée et Katia Vandenborre
}

\section{OpenEdition}

Journals

Édition électronique

URL : http://journals.openedition.org/slavica/70

DOI : 10.4000/slavica.70

ISSN : 2034-6395

\section{Éditeur}

Université libre de Bruxelles - ULB

Édition imprimée

Pagination : 22-30

ISSN : 2031-7654

\section{Référence électronique}

Aleksey V. Yudin, "La mythification des professions dans la culture traditionnelle des Slaves de l'Est », Slavica bruxellensia [En ligne], 1 | 2008, mis en ligne le 15 octobre 2008, consulté le 01 mai 2019. URL http://journals.openedition.org/slavica/70 ; DOI : 10.4000/slavica.70

\section{Ce document a été généré automatiquement le 1 mai 2019.}

\section{(c) (i) (9)}

Les contenus de Slavica bruxellensia sont mis à disposition selon les termes de la Licence Creative Commons Attribution - Pas d'Utilisation Commerciale - Pas de Modification 3.0 France. 


\title{
La mythification des professions dans la culture traditionnelle des Slaves de l'Est
}

\author{
Aleksey V. Yudin \\ Traduction : Anne Delizée et Katia Vandenborre
}

\section{NOTE DE L'ÉDITEUR}

Cet article a initialement été écrit en russe.

1 Le présent article est consacré à la mythification de certaines professions et plus généralement de certains types d'activité dans la culture traditionnelle des Slaves de l'Est. En d'autres termes, il étudie l'attribution par le peuple de certains savoir-faire, connaissances, pratiques, pouvoirs, contacts et rapports, pouvant être qualifiés de surnaturels, aux représentants de ces professions (prêtre, sorcier/guérisseur, meunier, forgeron, poêlier, apiculteur, pêcheur, chasseur, charpentier, vacher, potier, voleur, brigand, soldat). Notons qu'il existe déjà un article de T. V. Civ'jan sur une problématique similaire $^{1}$. Celui-ci rassemble un ensemble de données folkloriques et ethnographiques concernant les sorcières, les devins, les guérisseurs, etc. Sans répéter ce travail, ni en reprendre les exemples, nous nous proposons de quelque peu compléter le matériel de Civ'jan.

2 Étant la plus proche du monde du sacré, la figure du prêtre devrait être, semble-t-il, la plus mythifiée des figures de la vie rurale et paysanne. Il est vrai que non seulement les gens prêtaient des propriétés magiques aux sacrements accomplis par les prêtres, mais, en plus, ils craignaient toute rencontre fortuite avec un membre du clergé ou un moine ${ }^{2}$ (ce qui était partout considéré comme de mauvais augure) ${ }^{3}$. À ces exceptions près, apparemment, les prêtres et plus généralement le clergé ne faisaient toutefois pas tellement l'objet de mythification. Par ailleurs, d'après certains témoignages, les prêtres pouvaient se faire passer pour des sorciers auprès du peuple et plus particulièrement pour des exorciseurs de diables ${ }^{4}$. Cette dernière fonction était manifestement liée à une 
pratique, existante dans la religion orthodoxe, celle dite de vycityvanie (soustraction) du démon qui s'est emparé d'un homme (variante de l'exorcisme)5. En effet, il est relativement difficile de tracer une frontière entre la formule magique et la prière ; au sein du peuple, elle n'existait pas du tout ; c'est pourquoi il n'était pas rare qu'aux XVII et $\mathrm{XVIII}^{\mathrm{e}}$ siècles les copistes et les moines chargés de la diffusion orale et écrite des formules magiques fussent des membres du bas clergé (comme en témoignent les documents relatifs aux procès de sorcellerie).

3 Force est de constater que la figure la plus mythifiée était précisément celle du sorcier ${ }^{6}$. En tant qu'homme lié aux forces surnaturelles et capable "par leur intermédiaire d'influer sur la vie et l'activité des gens $»^{7}$, il était aussi un "professionnel» dans son genre. Habituellement, le sorcier « héritait» des pouvoirs magiques d'un vieux sorcier mourant ou alors devenait sorcier «de sa propre volonté, mais avec l'aide de l'esprit malin : "ils le [le malin] fréquentaient et, lui, il leur transmettait sa force" $»^{8}$. Avant de mourir, les sorciers souffraient tant qu'ils n'avaient pas légué leur pouvoir à quelqu'un d'autre' . Leur apparence extérieure, elle aussi, était inhabituelle :

Lors d'une conversation, le sorcier ne regarderait jamais son interlocuteur dans les yeux car, dans ses yeux, il n'y avait nul reflet. C'est ainsi que les sorciers se distinguaient. (...) Les commères disaient des yeux du sorcier qu'ils étaient vides, " bez mal'čikov"(sans garçon) $»^{10}$

(à ce propos, le vocable populaire «mal'čiki » pour désigner le reflet dans les pupilles est à l'origine d'un célèbre vers du monologue de Godunov dans le texte de Puškin: «Et les garçons sanglants dans les yeux »). Les sorciers étaient sorciers « de nature » (c'est-à-dire sorciers de naissance) ou « savants » (ayant acquis leurs connaissances et leur savoir-faire au cours de leur vie). Les premiers se distinguaient par la petite queue dont ils étaient pourvus, tout comme les sorcières. À la différence des guérisseurs, les sorciers étaient craints.

Il ne fallait pas être en mauvais termes avec un sorcier car tous les travaux domestiques et champêtres du paysan ainsi que son quotidien dépendaient de lui. Combattre un sorcier n'était possible qu'avec l'aide d'un autre sorcier, c'est pourquoi il était nécessaire de s'assurer le soutien de l'un d'entre eux. ${ }^{11}$

L'idée qu'un sorcier pouvait briser un mariage était répandue partout. Dans certaines régions, on considérait que l'on ne pouvait pas célébrer un mariage sans inviter un sorcier. En effet, celui-ci devait « le préserver des intrigues du démon et des dommages d'un autre sorcier qui obéirait au souhait malveillant d'habitants du même village, issus le plus souvent de la famille du fiancé éconduit ou de la fiancée abandonnée avant le mariage $»^{12}$.

5 Mais le sorcier n'était pas nécessairement, comme le veut le stéréotype, un sombre vieillard, vivant à l'écart et pratiquant la magie noire. Dans un sens plus large, par « sorcier » on entendait tous les gens qui détenaient des pouvoirs magiques bien définis, connaissaient les textes et se servaient de pratiques magiques dans leur activité professionnelle. Ces sorciers n'étaient pas des sorciers « de nature »:

Ils détenaient leur nature et leurs pouvoirs magiques, et plus précisément leur savoir professionnel, non pas génétiquement, ni par hasard (par imprudence, à cause de la perfidie d'un sorcier, etc.), mais avaient consciemment choisi leur voie, étudié et lu la littérature adaptée (nécromancie), etc. ${ }^{13}$

Chez les Slaves de l'Ouest et de l'Est, on considérait qu' étaient le plus souvent sorciers des gens de professions bien définies: les bergers, les forgerons, les meuniers, les apiculteurs, les chasseurs, les musiciens, etc. $»^{14}$ Civ'jan affirme que ces professions, « si 
l'on peut dire ainsi, prédisposaient à être sorcier ${ }^{15}$. Des connaissances mystérieuses et des relations particulières avec l'esprit malin étaient partout attribuées aux représentants des professions mentionnées. Par exemple, «selon la croyance populaire [de Vladimir], les meuniers, les apiculteurs et les pêcheurs concluaient des accords avec le génie des eaux $»^{16}$.

7 Les meuniers étaient généralement considérés comme des gens qui jouissaient de la protection particulière des génies des eaux ${ }^{17}$. Les génies des eaux (dans ces cas, ils étaient appelés mel'ničnye [génie des moulins]) vivaient surtout en eau trouble, dans les roues attenantes aux moulins, « sous les écluses, les barrages, les meules du moulin, voire dans les vieux moulins abandonnés,... Selon les croyances populaires, quand le moulin fonctionnait à plein régime, le génie des eaux était assis en haut de la roue et l'aspergeait d'eau.... Autrement dit, il mettait en mouvement la roue du moulin $»^{18}$. Il pouvait même réparer le moulin. Mais si le meunier se disputait avec lui, il se vengeait :

Il ouvrait les écluses, il faisait tourner les roues et les meules inutilement, démolissait complètement le moulin, le barrage, la digue et ne permettait pas d'en reconstruire de nouveaux. Les meuniers lui sacrifiaient des animaux morts, jetaient du pain dans le tourbillon, y versaient de la vodka et, en automne, mettaient sous la roue du moulin un morceau de saindoux ou un morceau de boyaux de porc afin que le génie des eaux ne dégraisse pas les roues du moulin en les léchant. ${ }^{19}$

Sous le pas de la porte du moulin, on enterrait vivant un coq noir et trois doubles tiges de rouille; on gardait au moulin des animaux de couleur noire (en règle générale, un coq et un chat), on portait sur soi de la laine de bouc noir, etc. Selon les croyances populaires, pour que le moulin soit prospère, il fallait promettre et offrir un ou plusieurs hommes. C'est pourquoi on disait des meuniers qu'ils attiraient les passants et les poussaient dans le tourbillon ou sous la roue du moulin. D'après la croyance populaire, si le meunier n'apportait pas l'offrande prévue, il était condamné à se noyer dans les plus brefs délais. ${ }^{20}$

8 Grâce au concours du génie des eaux, le meunier en personne acquerrait des pouvoirs magiques, qui lui permettaient d'aider ou de nuire aux gens ${ }^{21}$. D'après certains récits, le meunier rendait visite au génie des eaux, celui-ci à son tour allait chez le meunier et courtisait la femme du meunier sous l'apparence d'un beau garçon ${ }^{22}$. Le mécanisme du moulin à vent était aussi perçu comme « quelque chose de mauvais » lié à la sorcellerie ${ }^{23}$ : les propriétaires du moulin connaissaient, par exemple, des rituels pour attirer le vent.

De bons rapports avec le génie des eaux étaient tout aussi importants pour les pêcheurs. Selon eux, le génie des eaux «menait le poisson d'un endroit à l'autre et parfois le faisait sortir du filet $\aleph^{24}$. Dans le Nord russe, les pêcheurs apportaient au génie des eaux des offrandes spéciales: « Deux ou trois petits poissons, des miettes de pain, une cruche avec un fond de vin, une pincée de tabac, etc.", et parfois même un épouvantail anthropomorphe ${ }^{25}$. En général, ils lui sacrifiaient tout: depuis les animaux (un cochon noir ou un cheval volé par exemple) jusqu'au sel et au tabac. Les Pomores ${ }^{26}$ jetaient ce dernier dans l'eau en jurant. En effet, les vieux croyants estimaient que " puisque le tabac et les injures étaient des fruits de "l'impur", ils devaient être pour lui des présents agréables $»^{27}$. Les objets offerts au génie des eaux pouvaient même comprendre des laptis (espadrilles en tille) avec des chaussettes traditionnelles, cadeaux alors accompagnés de la formule magique suivante: "Prends les laptis, Diable, et rabats poisson dans nos filets! $\aleph^{28}$ Pour les Pomores, un vrai pêcheur se devait de connaître des formules pour que le poisson tombât dans son filet et les prononçait au moment de tendre le filet ${ }^{29}$.

Le pêcheur pouvait conclure un accord avec le génie des eaux. Celui-ci s'engageait alors à fournir à l'homme de nombreux poissons et à se mettre à son service. Mais 
les conditions étaient draconiennes : le pêcheur devait jeter un pendentif en croix dans l'eau et renier ses proches. Un tel pêcheur était condamné à se noyer tôt ou tard et son âme était mise à la disposition du démon..$^{30}$ pêche. Par exemple, «dans la province d'Arkhangelsk, avant le début de la pêche, les pêcheurs, regroupés en artel ${ }^{31}$,coupaient une miche de pain en morceaux (appelés šahmači ) et les jetaient dans le filet. Ensuite, ils traînaient le filet sur la glace sur une courte distance. Après quoi, l'ataman ${ }^{32}$ retirait les šahmači du filet et les distribuait aux pêcheurs. Les pêcheurs s'asseyaient autour du filet et mangeaient le pain, comme s'il s'agissait de pêche $»^{33}$. En naviguant au-dessus de l'endroit supposé être la demeure du génie des eaux, les pêcheurs et les passeurs enlevaient leur bonnet.

11 À minuit, la veille de « leurs » fêtes (par exemple à la « Fête du Sauveur du miel » (medovyj Spas $)^{34}$ ou à la Saint Zosime et Sabbatios de Solovki ${ }^{35}$ ), les apiculteurs faisaient, eux aussi, des offrandes au génie des eaux sous forme de rayons de miel. Le but était d'assurer le succès de leur production ${ }^{36}$, et surtout d'empêcher le génie des eaux d'inonder les ruches, qui souvent se trouvaient dans des prés au bord de l'eau, et d'envoyer aux abeilles de l'humidité, ce qui leur est néfaste ${ }^{37}$. Le génie des eaux était considéré comme le protecteur des abeilles. Selon les croyances populaires, la première colonie d'abeilles «avait essaimé d'un cheval épuisé par le génie des eaux. (...) Il était d'usage que, si l'apiculteur concluait un accord avec le génie des eaux, il lui faisait présent d'un coucou qu'il convenait de mettre dans une ruche à part. Tant que le coucou vivait chez l'apiculteur, ses abeilles lui donnaient beaucoup de miel. Mais il suffisait de le faire sortir pour que tout l'essaim prenne son envol et suive sa trace $»^{38}$. Aussi les apiculteurs protégeaient-ils leurs abeilles à l'aide de longues formules magiques, souvent adressées aux protecteurs de l'apiculture, Zosime et Sabbatios de Solovki (qui, d'après la légende, sont les premiers à avoir élevé des abeilles sur les îles Solovki).

Dans la représentation populaire, les forgerons eux aussi pactisaient avec le démon, et plus particulièrement avec les dragons de feu qui volaient dans le ciel. En règle générale, les gens soupçonnés de liens avec l'impur les gens qui exerçaient une profession qui les obligeait de vivre à l'écart, comme les forgerons. Ils étaient réputés être liés au diable, de la même façon que le meunier avec le génie des eaux ou le berger avec le lešij ${ }^{39}$. Dans les contes, le forgeron pouvait reforger la voix d'un personnage ou ferrer le diable.

13 Dans la représentation populaire, les potiers étaient eux aussi mythifiés. Dans les nombreuses bylick $i^{40}$, qui leur sont consacrés, il n'était pas rare que les potiers y rencontrassent un $"$ mort marchant $»^{41}$, apprissent leur métier chez des sorciers et devinssent eux-mêmes des sorciers. La richesse des potiers était attribuée à l'aide de l'esprit malin $^{42}$. "Dans la vaisselle d'un potier ne connaissant pas les secrets professionnels ${ }^{43}$, la crème fraîche ne prendra pas. ${ }^{44}$ Ils pouvaient même fabriquer des pots destinés à prendre de façon magique du lait chez les vaches d'un autre! Les pouvoirs surnaturels des potiers reposaient sans doute sur leur lien étroit avec le feu qui passait pour un objet de culte et qui, à l'époque chrétienne, pouvait être associé à la flamme de l'enfer ${ }^{45}$. Malgré les idées répandues concernant l'ivrognerie des potiers, cette profession jouissait, au sein du peuple, d'un respect considérable ${ }^{46}$.

14 Avant d'entamer une construction, les charpentiers accomplissaient certains rituels et faisaient un sacrifice dit "de construction": «Dans les fondations du bâtiment, ils mettaient un homme ou un animal. $»^{47}$ Selon la représentation populaire, les charpentiers qui, rassemblés en artel, allaient de village en village pour trouver du travail, maitrisaient 
l'art de la magie, connaissaient les formules magiques et étaient capables de les utiliser. Comme n'importe quels "étrangers ", ils étaient soupçonnés de rapports avec l'esprit malin. Pour se venger d'une offense (non-paiement par exemple), ils pouvaient par exemple introduire dans la maison une kikimora ${ }^{48}$, qui allait bruire et gronder toutes les nuits ${ }^{49}$, "renverser les meubles, mettre les planchers et les fourneaux sens dessus dessous, lancer des briques et d'autres objets sur les habitants... $»^{50}$. Nous citerons ici un récit de Vologda ${ }^{51}$ sur la sorcellerie des charpentiers.

Il était un moujik, dont on construisait la nouvelle maison... Quand elle fut entièrement bâtie, le maître de chantier vint chez le propriétaire pour régler les comptes. Le propriétaire les régla intégralement, mais voilà que l'ouvrier se mit à demander de la bière et un poluštof ${ }^{2}$ de vodka. Le propriétaire refusa. Les charpentiers s'en allèrent. Le propriétaire envoya son fils dans la nouvelle maison pour jeter un œil à la construction. Quand celui-ci entra, une petite souris bondit soudain, puis une autre plus grosse, puis encore une autre encore plus grosse, elles se mirent même à courir après le chat. Le fils prit peur et s'en retourna chez son père. (...) Le vieillard accourut auprès de son fils et cria : «Attelle vite un cheval et va chercher le contremaitre ». Le fils attela rapidement, prit de l'argent pour la vodka et s'en alla chez le contremaître, qu'il invita chez eux dans la nouvelle maison pour y pendre la crémaillère... Le vieillard reçut le contremaître avec du pain et du sel et le pria d'entrer dans la nouvelle maison. Tout à coup, une petite souris apparut et le contremaître lui dit : «Dis à ton troupeau qu'il décampe sur-lechamp!». En un instant, toutes les souris, les petites, les grandes, sortirent de l'isba. ${ }^{53}$

15 Les charpentiers pouvaient «simplement faire en sorte qu'il ne fût pas confortable de vivre dans la maison : la maison était traversée de toutes parts par des courants d'air, le poêle fumait, les murs chancelaient, etc. ${ }^{54}$

On considérait que pour construire un bon poêle, le poêlier devait prononcer les incantations appropriées ${ }^{55}$. D'autre part, offensé par le propriétaire, il pouvait «confectionner le poêle de façon à provoquer des bruits surnaturels: coups, grondements, hurlements, etc. ${ }^{56}$; pour ce faire, ils emmuraient divers objets dans la cheminée. En ce sens, les récits sur les poêliers sont très semblables à ceux sur les charpentiers. Le peuple racontait des choses à peu près similaires sur les maçons. C'est pourquoi les clients des représentants de ces professions les choyaient et réglaient toujours l'intégralité de leurs comptes.

17 La profession de vacherpouvait, dans certaines localités de Russie (à la différence de l'Ukraine), être perçue comme "humiliante et digne de mépris $~_{57}$. Par contre, dans le Nord russe (dans les régions d'Arkhangelsk et d'Olonetsk), ces gardiens de troupeau étaient respectés et même craints, car ils étaient considérés comme des sorciers associés aux lešij ${ }^{58}$ (esprit des forêts).

Le peuple croyait que le vacher concluait un contrat avec le lešij, qui en vertu de cet accord s'engageait à protéger le troupeau. En échange, le lešij recevait en été deux ou trois vaches ou du lait d'un, de deux, voire de trois trayons d'une vache... Malgré ce contrat, certains meneurs de bétail trompaient le lešij, en ne lui payant qu'un demi-oeuf de poule : ils en mangeaient une moitié et donnaient l'autre au lešij. ${ }^{59}$

Le vacher pouvait également prendre certains engagements envers le lešij: «ne pas manger de baies rouges ou noires, ne pas cueillir de champignons, ne pas se faire couper les cheveux, ni se raser, ne serrer la main à personne, ne pas se marier, ne pas jouer du garmoška $a^{60}$, ne passer par-dessus les haies ou encore d'autres choses de ce genre ${ }^{61}$. Cet accord était gardé secret. 

qui ne savaient pas », c'est-à-dire ceux qui maîtrisaient et ceux qui ne maîtrisaient pas la magie. Le travail des premiers était bien mieux rémunéré et les gens leur confiaient plus volontiers leur bétail. Le berger « qui savait» devait avant tout connaître le texte écrit d'une formule magique particulière, appelée "l'envoi du vacher ${ }^{62}$. Ces textes étaient d'ordinaire très longs et contenaient de nombreuses adresses à toutes les forces saintes et célestes, appelées à protéger le bétail. Le vacher prononçait habituellement la formule magique en tournant autour du troupeau, avant de les guider vers les pâtures ${ }^{63}$. Les rituels du premier pâturage sont également connus et assez compliqués. D'après des informations venant des régions de Vologda et d'Olonetsk, le vacher "prononçait une longue formule magique (...) et poussait le bétail entre des feux. Ensuite, il prenait du cérumen chez chaque animal qu'il roulait dans de la cire, un cadenas avec la clé, une motte de terre de terre provenant d'une tombe, un peu de terre d'une fourmilière se trouvant au carrefour de quatre chemins, une hache, un couteau et il poussait le bétail entre ces objets. Ensuite, il les enterrait dans le champ de pâture et les laissait là jusqu'à la fin du pacage estival du bétail. $»^{64}$

D'après les croyances populaires, les chasseurs pouvaient pactiser avec le lešij (en ayant renié au préalables les valeurs chrétiennes sacrées à l'aide de rites spéciaux. Pour ce faire, il convenait par exemple de tirer sur les Svjatye Dary ${ }^{65}$ ou de décharger son fusil en direction du son de la cloche de l'église. L'accord passé entre le chasseur et le lešij pouvait être écrit ou scellé par le sang ${ }^{66}$. Le lešij annonçait oralement les conditions de "l'accord " (quand, quoi et combien on pouvait obtenir) ou lui délivrait même un document spécial. L'esprit des forêts, lui, devait pousser le gibier dans les pièges ou encore assurer la justesse du coup de fusil. Le contrat supposait certaines conditions :

Par exemple, ne pas prendre plus de gibier que ce qu'il n'était permis, aller à la chasse des jours bien précis ou encore d'autres choses de ce genre. Dans le cas contraire, le lešij pouvait fouetter le chasseur avec les plus hautes branches des arbres, lui inoculer une maladie ou même le paralyser. ${ }^{67}$

Le chasseur, tout comme le vacher, était censé garder tout cela secret. En somme, sans cet accord, le chasseur n'attraperait rien de valable puisque c'est précisément le lešij qui partageait le butin entre les chasseurs et, sans sa permission, il n'était pas possible de tuer de bêtes. En ne respectant pas le contrat, le chasseur perdait les bonnes dispositions du lešij envers lui et, dans certains cas, il pouvait en mourir. À Pâques, les chasseurs, tout comme les vachers, allaient en forêt avec un œuf consacré pour échanger le baiser de Pâques avec le lešij ${ }^{68}$. Quand le chasseur avait l'intention de passer la nuit dans un abri en forêt, il était également d'usage de demander l'autorisation au lešij.

Les voleursaussi se servaient de pratiques magiques particulières, qui les aidaient à exercer leur métier de façon fructueuse. Par exemple, dévaliser quelqu'un dans l'église lors des mâtines pascales garantissait des vols réussis pendant toute l'année. Le peuple pouvait se représenter les brigands sous la forme de sorciers, surtout quand il s'agissait de brigands légendaires, de "nobles brigands $»^{69}$. On leur attribuait l'invulnérabilité, le pouvoir de se transformer en bêtes ou en oiseaux, d'ensorceler les armes, etc. ${ }^{70}$ Lorsqu'ils étaient jetés au cachot ou en prison, ils acquerraient le pouvoir d'échapper au gardien, d'endormir garde, de jeter un sort sur les fers. Des récits mythologiques étaient consacrés à Kudejar, l'ataman-brigand, à Stepan Razine, etc. Ce dernier, par exemple, était représenté sur un tapis volant (samoletka-samoplavka [qui vole tout seul-qui flotte tout seul]); il marchait dans le fond de la Volga comme sur la terre ferme, commandait les 
serpents, brisait ses fers grâce à l'Herbe fracassant ${ }^{71}$, etc. À Razine (comme à certains autres bandits) était attribué le pouvoir d'ensorceler les serpents et les grenouilles ${ }^{72}$.

Enfin, il faut encore citer un représentant du monde des «étrangers " qui pouvait être mythifié : le soldat. Dans les contes et les croyances populaires, il brisait souvent des charmes en s'opposant aux mauvais sorciers. Les soldats étaient capables d'ensorceler les fusils ou de se protéger par des sorts de la male mort ${ }^{73}$. À ce propos, on sait qu'il n'était effectivement pas rare que les soldats du tsar portassent dans des amulettes des textes de prières et de paroles magiques, devant les préserver de la balle ou de la lame ennemies. Ainsi, nous constatons que la mythification portait essentiellement sur les personnes qui se trouvaient en quelque sorte en dehors de la société paysanne étant donné leur profession ou leur façon de vivre, et qui étaient souvent en outre des intrus, des hommes venus d'autres villages ou d'autres contrées. Ceci est tout à fait naturel dans le cadre de la vision traditionnelle du monde, qui perçoit l'étranger avant tout comme un ennemi suscitant le doute et la suspicion. Les étrangers venus d'autres pays et/ou les hétérodoxes pouvaient également être mythifiés ${ }^{74}$. il est à noter que les mécanismes de la xénophobie actuelle diffèrent très peu des mécanismes traditionnels, ce qui pourrait faire faire l'objet d'une étude à part entière.

\section{BIBLIOGRAPHIE}

Civ'jan T. V., Ob odnom klasse personažej nizšej mifologii : "professionaly " (A propos d'une classe de personnages de la mythologie inférieure : « les professionnels »), in : Slavjanskij i balkanskij foL'klor. Narodnaja demonologija (Le folklore slave et balkanique. Démonologie populaire), Moscou, 2000, pp. 177-192.

Byt velikorusskih krest'jan-zemlepašcev. Opisanie materialov ètnografičeskogo bjuro knjazja V. N. Teniševa (Na primere Vladimirskoj gubernii) (Le Mode de vie des paysans et des laboureurs grand-russes. Description des documents du bureau ethnographique du prince V. N. Tenišev. [Sur le modèle de la province de Vladimir]), Firsov B. M. \& Kiseleva I. G. (réd.), Saint-Pétersbourg, 1993, p. 151.

Vlasova M. N., Russkie sueverija : Énciklopedičeskij slovar' (Les Superstitions russes : Dictionnaire encyclopédique), Saint-Pétersbourg, 2002

Tolstoj N. I., Slavjanskie drevnosti : Ėtnolingvističeskij slovar' (Les Antiquités slaves : Dictionnaire ethnolinguistique), t. 2 (D-K), Moscou, 1999

Bernštam T. A., Russkaja narodnaja kuL'tura Pomor'ja v XIX - načale XX v. Ėtnografičeskie očerki. (La Culture populaire russe du Pomore aux XIX et début du XX ${ }^{\mathrm{e}}$ siècle. Etudes ethnographiques), Saint-Pétersbourg (Leningrad), 1983, p. 185.

Kriničnaja N. A., Russkaja narodnaja mifologičeskaja proza: Istoki i polisemantizm obrazov : $v 3$ t. (La Prose mythologique populaire russe : Origines et polysémantisme des images : en 3 t.), t. 1 : Bylički, byvalščiny, legendy, pover'ja o duhah-“hozjaevah” (t. 1 : Bylines, histoires, légendes, croyances populaires sur les esprits-“maîtres"), Saint-Pétersbourg, 2002

Levkievskaja E. E., Mify russkogo naroda (Les mythes du peuple russe), Moscou, 2002 
Šaparova N. S., Kratkaja enciklopedija slavjanskoj mifologii (Petite encyclopédie de la mythologie slave), Moscou, 2002

Zelenin D. K., Vostočnoslavjanskaja etnografija (Ethnographie des Slaves de l'est), trad. de l'allemand par K. D. Civina, Moscou, 1991, p. 107.

Toporkov A. L., Gončarstvo : mifologia i remeslo (Poterie : mythologie et métier), p. 41, in : FoL'klor i ètnografija. U ètnografičeskih istokov fol'klornyh sjužetov i obrazov : Sbornik naučnyh trudov (Folklore et ethnographie. Aux sources des sujets et des images folkloriques : recueil de travaux scientifiques), Putilov B. N. (réd.), Saint-Pétersbourg (Leningrad), 1984

Moroz A. B., Severno-russkie pastušeskie otpuska i magija pervogo vygona skota u slavjan (Les Envois des vachers du Nord russe et la magie du premier pâturage du bétail chez les Slaves), in :

Vostočno-slavjanskij ètnoling-vističeskij sbornik. Issledovanija i materialy. (Recueil ethnolinguistique des Slaves de l'ouest. Recherches et documents), Moscou, 2001

Belova O. V., Poljaki i vostočnye slavjane : ètnokulturnye stereotipy v Poles'e (Les Polonais et les Slaves de l'est : les stéréotypes ethnoculturels en Polésie), in : Polacy w oczach Rosjan - Rosjanie w oczach Polaków. Poljaki glazami russkih - russkie glazami poljakov. Zbiór studiów (Les Polonais avec les yeux des Russes - les Russes avec les yeux des Polonais. Recueil d'études), Bobryk R i Faryno J. (réd.), Varsovie, 2000, pp. 59-65.

\section{NOTES}

1. Civ'jan T. V., Ob odnom klasse personažej nizšej mifologii : «professionaly " (A propos d'une classe de personnages de la mythologie inférieure : «les professionnels »), in : Slavjanskij i balkanskij foL'klor. Narodnaja demonologija (Le folklore slave et balkanique. Démonologie populaire), Moscou, 2000, pp. 177-192.

2. Il faut noter que, dans les formules magiques russes contre l'envoûtement, il est souvent fait mention de černec (moine), černuca (nonne) et plus rarement popapostrižnik (littéralement, celui qui est tonsuré : le moine) dans les listes de ceux qui pouvaient potentiellement jeter un sort sur un envoûté.

3. Byt velikorusskih krest’jan-zemlepašcev. Opisanie materialov ètnografičeskogo bjuro knjazja V.N. Teniševa (Na primere Vladimirskoj gubernii) (Le mode de vie des paysans et des laboureurs grandrusses. Description des documents du bureau ethnographique du prince V. N. Tenišev. [Sur le modèle de la province de Vladimir]), sous la direction de Firsov B. M. \& Kiseleva I. G., SaintPétersbourg, 1993, p. 151.

4. Vlasova M. N.., Russkie sueverija : Énciklope-dičeskij slovar' (Les superstitions russes : Dictionnaire encyclopédique), Saint-Pétersbourg, 2002, p. 253 ; Civ'jan T.V., Art.cit., Moscou, 2000, pp. 177-192.

5. A cet égard le témoignage suivant, en provenance de la province de Toula, mérite d'être cité : «A Khmelniki, la moitié du village est composée de sorciers, tous sont des élèves du diacre décédé » : Vlasova M. N..., Ibid., p. 253.

6. Firsov B. M. \& Kiseleva I. G., Ibid., p.129-132; Tolstoj N. I., Slavjanskie drevnosti : Ėtnolingvističeskij slovar' (Les antiquités slaves : Dictionnaire ethnolinguistique), t. 2 (D-K), Moscou, 1999, p. 528-534 ; Vlasova M. N..., Ibid., pp. 242-258.

7. Bernštam T. A., Russkaja narodnaja kuL'tura Pomor’ja v XIX - načale XX v. Ėtnografičeskie očerki. (La culture populaire russe du Pomore aux XIX et début du XXe siècle. Etudes ethnographiques), Saint-Péterbourg (Leningrad), 1983, p. 185.

8. Firsov B. M. \& Kiseleva I. G., Ibid., p. 129.

9. Ibid., p. 132. 
10. Idem.

11. Bernštam T. A., Ibid., p. 185.

12. Ibid., p. 186.

13. Civ'jan T. V., Ibid., p. 179.

14. Tolstoj N. I., Ibid., p. 530 ; voir la liste de ces professions in : Civ'jan T. V., Ibid., p. 180-181.

15. Civ'jan T. V., Ibid.,p. 180.

16. Firsov B. M. \& Kiseleva I. G., Op. cit., p. 126.

17. Kriničnaja N. A., Russkaja narodnaja mifologičeskaja proza: Istoki i polisemantizm obrazov: $v 3$ t. (La prose mythologique populaire russe: Origines et polysémantisme des images: en 3 t.), t. 1 : Bylički, byvalščiny, legendy, pover'ja o duhah-“hozjaevah” (t. 1 : Bylines, histoires, légendes, croyances populaires sur les esprits-“maîtres"), Saint-Pétersbourg, 2002, p. 488.

18. Idem, p. 488-489.

19. Levkievskaja E. E., Mify russkogo naroda (Les mythes du peuple russe), Moscou, 2002, pp. 347-348.

20. Šaparova N.S.., Kratkaja enciklopedija slavjanskoj mifologii (Petite encyclopédie de la mythologie slave), Moscou, 2002, p. 356.

21. Kriničnaja N. A., Ibid., p. 490.

22. Vlasova M. N..., Ibid., p. 338.

23. Civ'jan T. V., Op. cit.., p. 180.

24. Zelenin D. K., Vostočnoslavjanskaja etnografija (Ethnographie des Slaves de l'est), trad. de l'allemand par K. D. Civina, Moscou, 1991, p. 107.

25. Idem.

26. Le terme « Pomore » signifie en russe « habitants des côtes » ou « peuple habitant près de la mer ». Il s'agit d'un peuple du Nord russe vivant sur le littoral de la mer Blanche et de la mer de Barents (NdT.).

27. Bernštam T. A., Ibid., p. 176.

28. Levkievskaja E. E., Ibid., p. 347.

29. Bernštam T. A., Ibid., p. 186.

30. Levkievskaja E. E., Ibid., p. 347.

31. L'artel est une coopérative de production dans l'ancienne Russie (NdT.).

32. L'ataman est un chef chez les Cosaques. Elu par ceux-ci, il exerce des fonctions politiques et militaires (NdT.).

33. Zelenin D. K., Ibid., p. 108.

34. Appellation populaire de la fête orthodoxe« Procession de la Sainte et Vivifiante Croix » à Constantinople qui se déroule le 1er/14 août (NdT).

35. Fête orthodoxe de la translation des Reliques, le $8 / 22$ août (NdT).

36. Kriničnaja N. A., Ibid., p. 494.

37. Levkievskaja E. E., Ibid., p. 348.

38. Idem.

39. Dans la mythologie russe, le lešij est un esprit démoniaque des bois et des forêts. A propos du rôle du double protecteur surnaturel dans la sacralisation des professions, voir: Civ'jan T.V., Op. cit., p. 183.

40. Brèves histoires folkloriques en prose (memorat) consacrées aux rencontres avec les forces impures et mettant en scène une personne qui n'est pas le conteur (NdT).

41. Toporkov A. L., Gončarstvo: mifologia i remeslo (Poterie: mythologie et métier), p. 41, in : FoL'klor i ètnografija. U ètnografičeskih istokov fol'klornyh sjužetov $i$ obrazov: Sbornik naučnyh trudov (Folklore et ethnographie. Aux sources des sujets et des images folkloriques : recueil de travaux scientifiques), sous la direction de Putilov B. N., Saint-Péterbourg (Leningrad), 1984, pp. 41-47. Le «Mort marchant » est fait référence à un mort qui quitte sa tombe sans influence magique de qui que ce soit pour faire le mal parmi les vivants.

42. Ibid., p. 42. 
43. Concernant les rituels et les formules magiques nécessaires à la fabrication de la vaisselle, mais aussi au temps de sa confection.

44. Toporkov A. L., Ibid., p. 42.

45. Ibid., pp. 43-44.

46. Ibid., pp. 44-45.

47. Kriničnaja N. A., Ibid., p. 276. Les sacrifices humains n'étaient toutefois pas des phénomènes courants chez les Russes.

48. Dans la mythologie russe, petit être invisible de sexe féminin, vivant essentiellement dans les maisons, derrière le poêle ; équivalent féminin du domovoj ( $\mathrm{NdT}$ ).

49. Civ'jan T. V., Ibid., p. 188.

50. Vlasova M. N.., Ibid., p. 222.

51. Vologda est une ville du nord-ouest de la Russie (NdT.).

52. Mesure russe équivalente à $0,76868750 \mathrm{l}$, c'est-à-dire à une bouteille de vin (NdT.).

53. Vlasova M. N.., Op. cit., p. 254.

54. Šaparova N. S.., Op. cit., p. 416. Nous trouverons ici un examen détaillé des désagréments que pouvaient causer les charpentiers offensés aux futurs propriétaires : ils pouvaient, en particulier, enfoncer un clou de cercueil sous un banc dans le Coin Rouge (Krasnyj ugol) là où étaientinstalléeslesicônes ;en conséquence de quoi, en entrant dans la maison, les membres de la famille voyaient dans ce coin un mort. Les charpentiers pouvaient aussi jeter un sort sur la nouvelle maison, provoquant ainsi la mort précoce du propriétaire et la ruine de sa maison.

55. Kriničnaja N. A., Op. cit.., p. 250.

56. Civ'jan T. V., Op. cit., p. 188.

57. Zelenin D. K., Op. cit., p. 87.

58. Idem.

59. Idem

60. Instrument de musique traditionnel russe : petit accordéon diatonique à boutons $(\mathrm{NdT})$

61. Levkievskaja E. E., Op. cit., p. 332.

62. Formule magique prononcée par le gardien du troupeau avant d'envoyer le bétail paître dans la forêt sans sa surveillance, afin d'éviter qu'il s'y perdre ou qu'il y soit dévoré par les prédateurs. Plus de détails in : Moroz A. B., Severno-russkie pastušeskie otpuska i magija pervogo vygona skota u slavjan (Les envois des vachers du Nord russe et la magie du premier pâturage du bétail chez les Slaves), in: Vostočno-slavjanskij ètnolingvističeskij sbornik. Issledovanija i materialy. (Recueil ethnolinguistique des Slaves de l'ouest. Recherches et documents), Moscou, 2001, pp. 232-258; Levkievskaja E. E., Op. cit., pp. 332-335.

63. Zelenin D. K., Op. cit., p. 87.

64. Ibid., p. 90. A propos de la sacralisation de la profession de berger, voir : Civ'jan T. V., Op. cit., p. 181 ; «Dossier mythologique» du berger, basé sur différentes traditions indo-européennes : Idem, pp. 183-184.

65. Saints Dons Présanctifiés, c'est-à-dire le Corps et le Sang du Christ, que le chasseur mettait en bouche sans avaler et recrachait une fois sorti de l'église (NdT).

66. Kriničnaja N. A., Op. cit., pp. 372-373.

67. Levkievskaja E. E., Op. cit., p. 331.

68. Kriničnaja N. A., Op. cit., p. 372.

69. Civ'jan T. V., Op. cit., p. 180.

70. Vlasova M. N.., Op. cit., p. 252.

71. Herbe magique, cueillie dans la forêt, qui a le pouvoir de briser les fers, ouvrir les serrures, casser les grilles de prison, etc. (NdT).

72. Idem.

73. Ibid., p. 253. 
74. Voir par exemple: Belova O. V., Poljaki $i$ vostočnye slavjane : ètnokulturnye stereotipy $v$ Poles'e (Les Polonais et les Slaves de l'est : les stéréotypes ethnoculturels en Polésie), in : Polacy w oczach Rosjan - Rosjanie w oczach Polaków. Poljaki glazami russkih - russkie glazami poljakov. Zbiór studiów (Les Polonais avec les yeux des Russes - les Russes avec les yeux des Polonais. Recueil d'études), sous la direction de Bobryk R. i Faryno J., Varsovie, 2000, pp. 59-65.

\section{AUTEURS}

\section{ALEKSEY V. YUDIN}

Docteur en philologie slave, enseignant au département de Slavistique et d'études de l'Europe de l'Est à l'Université de Gand (UGent, Belgique) 